\title{
Simultaneous growth of two cancer cell lines demonstrates variability in growth rates
}

This article was published in the following Dove Press journal:

Open Access Medical Statistics

28 November 2014

Number of times this article has been viewed

\author{
Agnes Hamon ${ }^{1-3}$ \\ Marie Tosolini ${ }^{3-6}$ \\ Bernard Ycart ${ }^{1-3}$ \\ Frederic Pont ${ }^{3-6}$ \\ Jean-Jacques Fournie ${ }^{3-6}$ \\ 'Université Grenoble-Alpes, \\ ${ }^{2}$ Laboratoire Jean Kuntzmann, \\ Grenoble, ${ }^{3}$ Laboratoire d'Excellence, \\ Toucan, ${ }^{4}$ INSERM UMR I037, Cancer \\ Research Center of Toulouse, \\ Toulouse, ${ }^{5}$ Université Toulouse III \\ Paul-Sabatier, Toulouse, ${ }^{6}$ ERL 5294 \\ CNRS, Toulouse, France
}

\begin{abstract}
Cancer cells cocultured in vitro show unexpected differential growth rates that classical exponential growth models cannot account for. Two noninteracting cell lines were grown in the same culture, and counts of each species were recorded at periodic intervals. The relative growth of population ratios was found to depend on the initial proportion, in contrast with the traditional exponential growth model. A proposed explanation is the variability of growth rates for clones inside the same cell line. This leads to a log-quadratic growth model that provides both a theoretical explanation for the phenomenon that was observed, and a better fit for our growth data.

Keywords: cancer cells, growth, log-linear model
\end{abstract}

\section{Introduction}

Given that emergence of resistant cells underlies the time to relapse for cancer patients undergoing chemotherapy, the growth rate of these tumor cells is a crucial issue. Cancer therapies usually yield undetectable levels of residual and resistant cancer stem cells (CSCs) in patients. However, upon repeated mitosis, CSCs can seed a cell progeny that progressively reconstitutes tumors, but the proportion and mitotic rate of such CSCs are highly variable in treated patients. The classical exponential growth model predicts that the relative growth of fast-growing clones should increase exponentially with time, regardless of their initial rates in patients. On the other hand, however, this model is challenged by heterogeneity of the clonal progeny from a cancer cell and the resulting Darwinian selection in this progeny for access to nutrients. ${ }^{1,2}$ To investigate this, we grew two noninteracting human cancer cell lines either separately or together in cell cultures with medium containing an unlimited supply of nutrients, and modeled the cell growth rates observed in the cocultures. The exponential growth model is so elementary and has been known for such a long time, ${ }^{3,4}$ that it seems almost too simple to actually fit real cell growth data. ${ }^{5}$ However, for a given cell line grown in unlimited supporting medium, an excellent linear fit is usually observed for the logarithm of population size against time. ${ }^{6,7}$ Our experiments were conducted with two well known laboratory strains, ie, a non-Hodgkin's lymphoma B-cell line (American Type Culture Collection [ATCC] CRL-2261; ${ }^{8} \mathrm{RL}$ ) and a cell line derived from a patient with acute monocytic leukemia (TCC TIB-202; ${ }^{9}$ THP-1). As a control experiment, the two cell lines were grown separately. An excellent log-linear fit was observed. Both strains were grown in the same solution. No interaction between the two species could occur, other than possible competition for nutrients in the culture medium. This was avoided by maintaining a sufficient supply of medium by volume and continuous renewal. The initial
Correspondence: Bernard Ycart

$5 \mathrm{I}$ rue des mathématiques,

Grenoble Cedex 3804I, France

$\mathrm{Tel}+334765 \mathrm{I} 4995$

Fax +33476631263

Email bernard.ycart@imag.fr 
proportions of the faster growing RL strain were fixed at $0.5 \%, 1 \%, 5 \%$, and three replicates were made for each initial proportion. With the classical exponential growth model, the relative proportion of RL versus THP-1 would be predicted to increase exponentially over time, at a rate independent of the initial proportions. Somewhat unexpectedly, this turned out to be false. Figure 1 presents a plot of the ratio of observed RL versus THP-1 counts on a logarithmic scale. The time scales have been shifted so that the origin corresponds to the time at which each proportion reaches $5 \%$. The slope of the regression line decreases as the initial proportion of the more rapidly growing strain increases, ie, the slope with an initial proportion of $5 \%$ (red) is smaller than that with initial proportions of $1 \%$ (green) and $0.5 \%$ (blue).

In vitro experiments with simultaneous growth of two or more microorganisms have been carried out for a long time (see Dykhuizen ${ }^{10}$ for a review). The variability of growth rates in human leukemia cell clones has been investigated by Tomelleri et al. ${ }^{11}$ However, to the best of our knowledge, this is the first description of an experiment with two different cancer lines showing the phenomenon of dependence of the ratio growth rate on the initial condition, as shown in Figure 1.

The objective of this paper is to propose a stochastic growth model explaining this phenomenon, and show that the fit of the data by that model is better than that with the exponential growth model.

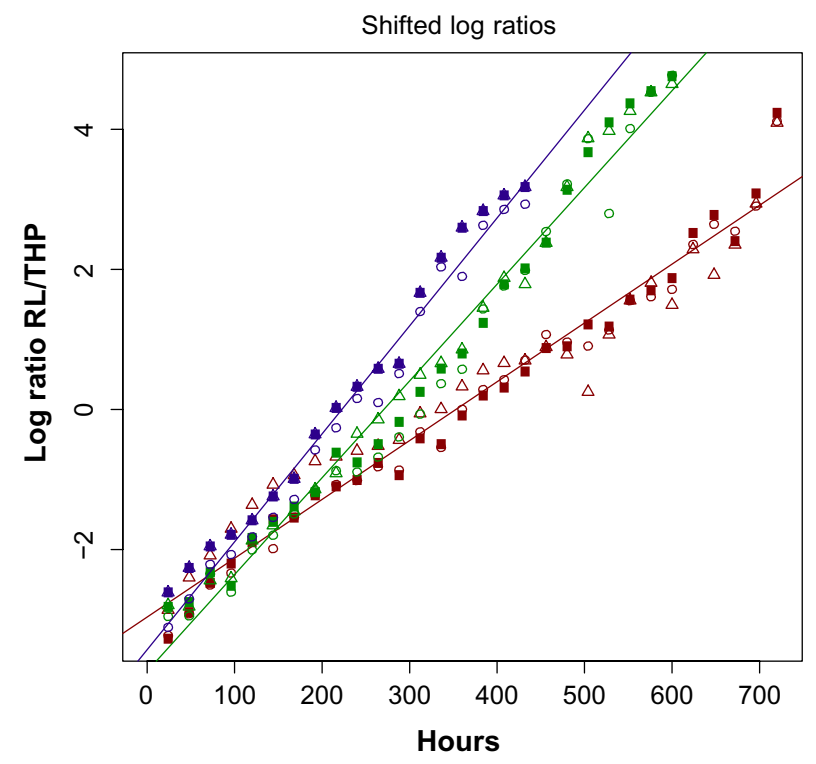

Figure I Logarithms of ratios of RL/THP-I. The three replicates for each initial condition are marked by circles, triangles and squares. All curves have been shifted to the first day where the proportion RL/THP-I passes $5 \%$. Red marks correspond to an initial proportion of $5 \%$, green marks to $1 \%$, blue marks to $0.5 \%$. The three regression lines are represented with corresponding colors. The slopes (time unit: hour) are 0.0084 (red line, initial proportion 5\%), 0.0138 (green line, initial proportion $1 \%$ ), and 0.0154 (blue line, initial proportion $0.5 \%$ ).
It has long been known that exponential proliferation is a valid approximation only for a certain fraction of the observation period. ${ }^{12}$ Many different models have been proposed as growth curves. ${ }^{13,14}$ At the beginning of a cell growth experiment, a lag phase ${ }^{15-17}$ is usually observed, and this is the case in our data. This lag phase could account in part for the phenomenon investigated here. Indeed, when starting from a proportion of $0.5 \%$, the lag phase has elapsed when reaching $1 \%$, but if one starts with a $1 \%$ proportion, the lag phase only begins at the initial instant. However, the lag phase does not explain differential growth rates after all cultures have reached a proportion of $5.0 \%$, since at that time, the lag phase has elapsed in the first two cases. Another simple explanation is proposed here, ie, the intrinsic variability of growth rates. ${ }^{5,11}$ Here, the notion of growth rate is understood in the sense of branching processes ${ }^{4}$ as a "large-scale approximation" that applies to the whole clone stemming from a given cell and not just to that cell alone. Therefore, the variation in growth rates can only be genetic, ie, one value is associated with each cell present at the beginning of the experiment, and this will be the growth rate of the whole clone stemming from that cell. If growth rates among RL cells vary, the proportion of fast dividing mitotic cells among RL cell clones will gradually increase. When reaching the proportion of $5 \%$, there will be more rapid breeders among RL if the initial proportion was $0.5 \%$ than if it was $1 \%$. This intuitively explains why the estimated growth rate over a given time interval is larger when the proportion of RL reaches $5 \%$ after starting from $0.5 \%$.

Mathematically, it will be shown that, assuming variable growth rates among cells of the same species, naturally leads to a log-quadratic model of population growth instead of the traditional log-linear (or exponential) model. It will be shown that the log-quadratic model induces a better fit for our data. Using that model, the observed phenomenon can be explained and quantified. Indeed, if the actual growth is log-quadratic instead of log-linear, the fit of a log-linear model yields estimated slopes that vary with the initial condition; this theoretical explanation is shown in Figure 2. The derivation of the logquadratic model from the hypothesis of variable growth rates uses the cumulant generating function of random growth rates. A similar explanation had been proposed by Hansen. ${ }^{18}$

\section{Materials and methods}

\section{Experimental methods}

The THP-1 (ATCC TIB-202) cell line derives from human acute monocytic leukemia. It has a monocyte morphology and expresses the cell surface marker CD13. The cell line RL 


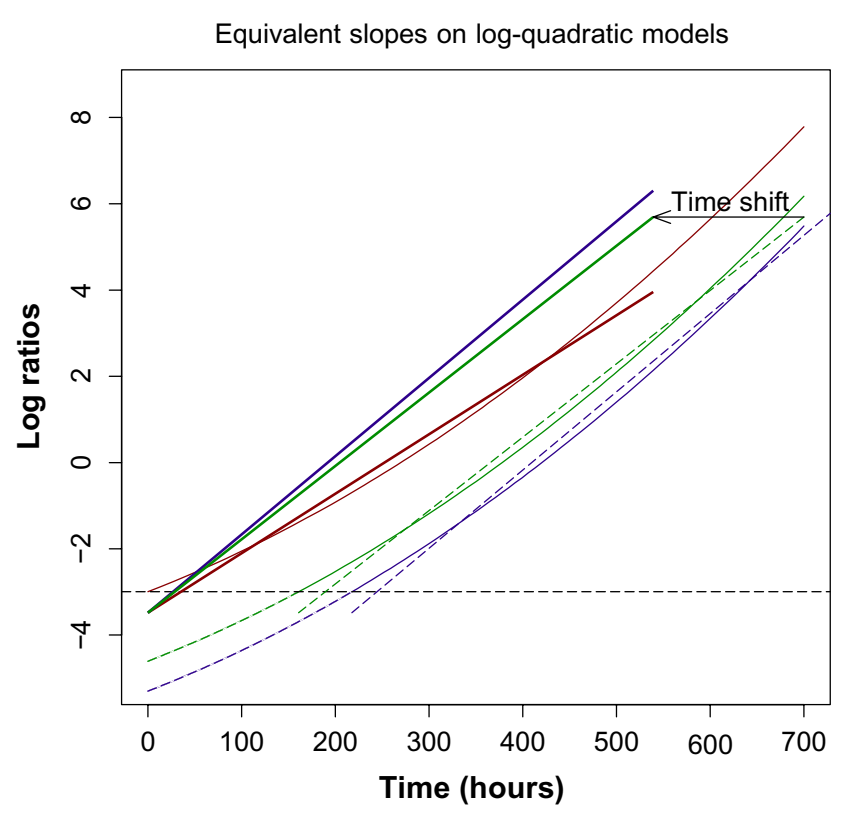

Figure 2 Theoretical explanation for observed differential growth rates. The figure represents a quadratic growth in time, at three different intercepts: $\log (0.05)$ (red), $\log (0.0 \mathrm{l})$ (green), and $\log (0.005)$ (blue). The three solid curves are parabolas. The green and blue dashed lines are linear fits over an interval starting at the point where the corresponding parabola reaches $\log (0.05)$. The green and blue solid lines are time shifts, illustrating the differential slopes.

(ATCC CRL-2261) was derived from human non-Hodgkin's lymphoma and expresses the cell surface marker CD20. These two cell lines were cultured as indicated by the supplier (ATCC, Manassus, VA, USA; http://www.lgcstandards-atcc. org) at $37^{\circ} \mathrm{C}$ and $5 \% \mathrm{CO}_{2}$ in liquid Roswell Park Memorial Institute-1640 medium (Lonza, Levallois, France) supplemented with $10 \%$ heat-inactivated fetal calf serum, $2 \mathrm{mM}$ L-glutamine, $100 \mathrm{U} / \mathrm{mL}$ penicillin, and $100 \mathrm{~g} / \mathrm{mL}$ streptomycin (Invitrogen, Cergy Pontoise, France). This medium contains inorganic salts, amino acids, vitamins, and D-glucose ( $2 \mathrm{~g} / \mathrm{L})$. THP-1 and RL cells, alone and in competition, were cultured in T75 flasks with $50 \mathrm{~mL}$ of medium without agitation. At the beginning of culture, cells were seeded at $0.3 \times 10^{6}$ cells $/ \mathrm{mL}$ per day for all conditions.

The different cell cultures were counted and, if necessary, diluted with complete medium if the cell concentration was higher than $0.7 \times 10^{6}$ cells $/ \mathrm{mL}$ to adjust the concentration to $0.3 \times 10^{6}$ cells $/ \mathrm{mL}$. These concentrations lead to no competition for nutrients. The culture dilutions were adjusted to the cell growth in each flask; eg, RL cell culture was more diluted than THP-1 cell culture. Cells from simultaneous cultures were analyzed by flow cytometry in the following manner to determine the percentage of each cell line in the culture. The cells were centrifuged, washed with phosphate-buffered saline and incubated for 10 minutes with antibodies against CD20 coupled with the fluorochrome APC-Cy7 to identify
RL cells and against CD13 coupled with the fluorochrome phycoerythrin (both from BD Biosciences, Pont de Claix, France) to identify THP-1 cells. Cells cultured from a single cell line were used as controls. The fluorescence of 50,000 cells was then analyzed using an LSR II cytometer (BD Biosciences).

Two sets of experiments were performed over 35 days, with daily measurements. In the first set, the two cell lines (RL and THP-1) were grown in separate culture flasks in duplicate. In the second set, the two cell lines were grown in the same culture flask in triplicate with an unlimited amount of nutrients in each case. Three initial proportions of RL (the more rapidly growing strain) were considered, ie, $0.5 \%, 1 \%$, and $5 \%$. For each set of experiments, each day of culture, each replicate, the numbers of cells of each type were recorded. The dataset is available upon request. For these data, different least square fits of the log-quadratic model (6) were performed for each of the two separate growth patterns (first set of experiments), and for simultaneous growth (second set of experiments).

\section{Mathematical model}

In this section, a mathematical derivation of a log-quadratic growth model, based on variable growth rates, is proposed. Consider first the classical model of exponential growth for a single clone, stemming from the general theory of branching processes. ${ }^{4,19,20}$ From a single cell at time 0 , the clone grows to size $N(t)$ at time $t$. Under fairly general hypotheses on the division time distribution, there exists a positive constant $b$, the growth rate (also called the Malthusian parameter), such that almost surely:

$$
\lim _{t \rightarrow+\infty} e^{-b t} N(t)=C,
$$

where $C$ is a random variable with finite expectation and variance. This is one of the basic results of the theory of branching processes. ${ }^{19,20}$ Thus, it is reasonable to assume $N(t)=C e^{b t}$ as a model of the growth curve for a single clone. Assume now that the population grows from a large number $n$ of identical initial cells.

For $i=1, \ldots, n$, let $N_{i}(t)$ be the size at time $t$ of the clone stemming from cell $i$ :

$$
N_{i}(t)=e^{b t} C_{i},
$$

where the $C_{i}$ are independent identically distributed random variables. The total population at time $t$ is:

$$
N(t)=\sum_{i=1}^{n} N_{i}(t) .
$$


By the law of large numbers, almost surely:

$$
\lim _{n \rightarrow \infty} \frac{N(t)}{n}=e^{v t} \mathbb{E}(C)
$$

where $\mathbb{E}(C)$ denotes the mathematical expectation of the random variable $C$. This justifies the classical log-linear model:

$$
\log (N(t))=a+b t
$$

where $a=\log (N(0))$. General references on log-linear models are given in Mair ${ }^{21}$ and von Eye and Mun. ${ }^{22}$

Consider now a second population growing according to the same model, and denote by $M(t)$ its size at time $t$.

$$
\log (M(t))=a^{\prime}+b^{\prime} t .
$$

Assume $b>b^{\prime}$ (the first population grows faster). The ratio $R(t)=N(t) / M(t)$ then also follows a log-linear model:

$$
\log (R(t))=\left(a-a^{\prime}\right)+\left(b-b^{\prime}\right) t
$$

Whatever the interval of time it is observed in, the growth rate $b-b^{\prime}$ does not depend on the interval nor on the initial proportion. This is contradicted by our observations (Figure 1).

Assume now that clones stemming from different initial cells may have different growth rates. The new model is:

$$
N_{i}(t)=e^{B_{i} t} C_{i},
$$

where $\left(B_{i}, C_{i}\right)$ are independent and identically distributed copies of a random couple $(B, C)$. The joint distribution of $(B, C)$ is of course unknown, and we shall make the technical assumptions that $B$ and $C$ are independent, and that $B$ has faster than exponential decaying tails. By the same argument of law of large numbers, the global population $N$ ( $t$ ) should satisfy:

$$
\log (N(t))=a+\log \left(\mathbb{E}\left(e^{B t}\right)\right)
$$

Note that the function $\log \left(\mathbb{E}\left(e^{B t}\right)\right)$ exists for all $t \geq 0$ if the distribution of $B$ has more rapid than exponential decaying tails. This function is the cumulant generating function of $B,{ }^{23}$ well known in large deviation theory. ${ }^{24}$ Let $\mu$ be the expectation of $B, \sigma$ its standard deviation, and $\gamma_{1}$ its skewness. The first three terms of the Taylor expansion of $\log \left(\mathbb{E}\left(e^{B t}\right)\right)$ then are:

$$
\log \left(\mathbb{E}\left[e^{B t}\right]\right)=\mu t+\frac{\sigma^{2}}{2} t^{2}+\frac{\sigma^{3} \gamma_{1}}{6} t^{3}+o\left(t^{3}\right),
$$

In the particular case where $B$ follows a Gaussian distribution, the first two terms give the exact expression:

$$
\log \left(\mathbb{E}\left[e^{B t}\right]\right)=\mu t+\frac{\sigma^{2}}{2} t^{2}
$$

In that case, the growth of $N(t)$ is quadratic in logarithmic scale:

$$
\log (N(t))=a+b t+c t^{2}
$$

with $\mathrm{a}=\log (N(0)), b=\mu$, and $c=\sigma^{2} / 2$. Equation (6) will be referred to as a log-quadratic model (see von Eye and Mun ${ }^{22}$ (Chapter 9) and Stone et $\mathrm{a}^{25}$ for an application in a similar context). Assuming that the distribution of $B$ is Gaussian may seem unrealistic, but whatever the distribution of $B$, if its expectation is $\mu=b$ and variance $\sigma^{2}=2 c$, equation (6) remains true as a second-order approximation because of (5). This justifies the use of (6) as a model, in case of variable growth rates.

If two populations grow according to a log-quadratic model, then the ratio of the two population sizes does too. Denote again that ratio by $R(t)$, assuming that the choice has been made to put the more rapidly growing population on the numerator, so that $R(t)$ increases.

$$
\log (R(t))=a+b t+c t^{2}
$$

with $a=\log (R(0))$ and $b, c>0$. In practice, growth rates are estimated by a log-linear regression over a given interval, say $\left[T_{1}, T_{2}\right]$. This amounts to approximating (7) by:

$$
\log (R(t))=\hat{\alpha}+\hat{\beta} t
$$

where $\hat{\alpha}$ and $\hat{\beta}$ are optimal in the sense of mean squares:

$$
(\hat{\alpha}, \hat{\beta})=\arg \min \int_{T_{1}}^{T_{2}}\left(a+b t+c t^{2}-\alpha-\beta t\right)^{2} d t .
$$

The solution of (8) is easily obtained:

$$
\hat{\alpha}=a-\frac{c}{6}\left(T_{1}^{2}+T_{2}^{2}+4 T_{1} T_{2}\right) \text { and } \hat{\beta}=b+c\left(T_{1}+T_{2}\right) .
$$

For a fixed span $T_{2}-T_{1}$, the "equivalent growth rate" $\hat{\beta}$ increases as $T_{1}$ increases (see Figure 2 for an illustration). This explains the phenomenon shown in Figure 1. More precisely, let $T_{1}$ be the time at which $R$ reaches the value $R_{1}>R(0)$ :

$$
T_{1}=\frac{1}{2}\left(-b+\sqrt{b^{2}+4 c \log \left(\frac{R_{1}}{R(0)}\right)}\right) .
$$

The equivalent growth rate in a time interval of duration $t$ after $T_{1}$ will be $\hat{\beta}=b+c\left(2 T_{1}+t\right)$. It will be larger than the growth rate during an interval of the same width starting at 0 , which is $b+c t$. 
Table I Estimations for the logarithm of RL cell counts

\begin{tabular}{llll}
\hline Coefficient & Estimate & SE & $P$-value \\
\hline$a$ & 12.21 & $3 \times 10^{-2}$ & $<2 \times 10^{-16}$ \\
$b$ & 0.0313 & $9 \times 10^{-5}$ & $<2 \times 10^{-16}$ \\
\hline
\end{tabular}

Abbreviations: SE, standard error; RL cells, American Type Culture Collection CRL-226I cells.

Thus the log-quadratic model (6) provides a theoretical explanation for the phenomenon of the differential growth rates observed. As will be shown in the next section, it also provides a better fit with our data.

\section{Results}

\section{Separate growth of RL}

Let $Y_{i k}$ denote the logarithm of cell count at time $t_{k}$ and replicate $i(i=1,2)$.

We consider the following model:

$$
Y_{i k}=a+b t_{k}+c t_{k}^{2}+\varepsilon_{i k}
$$

where $\varepsilon_{i k}$ are centered Gaussian random variables with a common standard deviation. For RL cells, it turned out that

Global fit

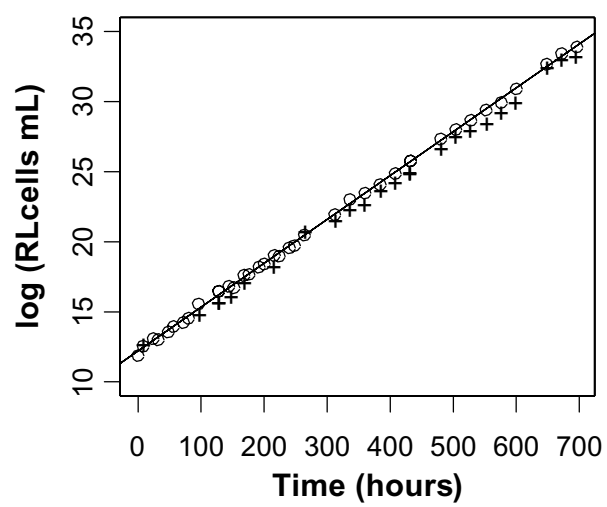

Residuals versus time

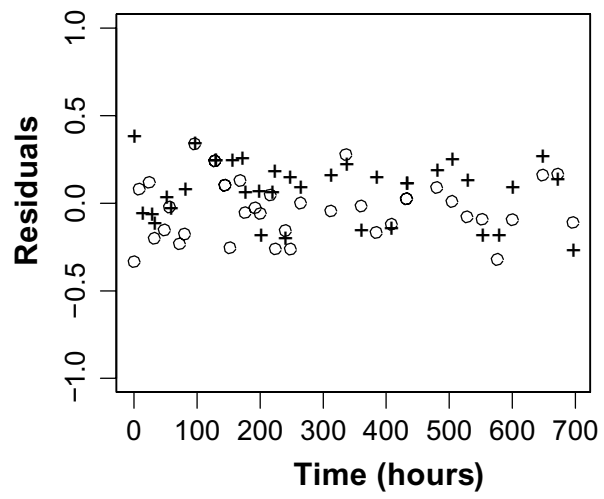

the coefficient $c$, which we shall call "curvature", was not significantly different from zero $(P=0.698)$. Therefore, a linear model without a quadratic term was fitted. Table 1 reports the estimated coefficients. Figure 3 presents the residual analysis. The two coefficients $a$ and $b$ are significantly different from zero. The $95 \%$ confidence interval of the mean $\mathrm{RL}$ growth rate $b$ is $[0.0311,0.0315]$. This corresponds to a doubling time between 22 and 22.3 hours. The proportion of the variation of $Y_{i k}$ explained by the fitted model is excellent $\left(R^{2}=0.99\right)$. The QQ plot of residuals (Figure 3$)$ is close to linear, and the plot of residuals versus time does not show any misspecification of the non-random part or a heteroscedasticity problem. The Durbin-Watson test $(P=0.12)$ and the runs test $(P=0.69)$ indicate no violation of the hypothesis of error independence.

\section{Separate growth of THP-I}

The model remains the same, see Equation (9). At first, it was fitted to the full dataset. Three observations at the end of the experiment period were detected as outliers, and therefore excluded from the final analysis. Table 2 reports the
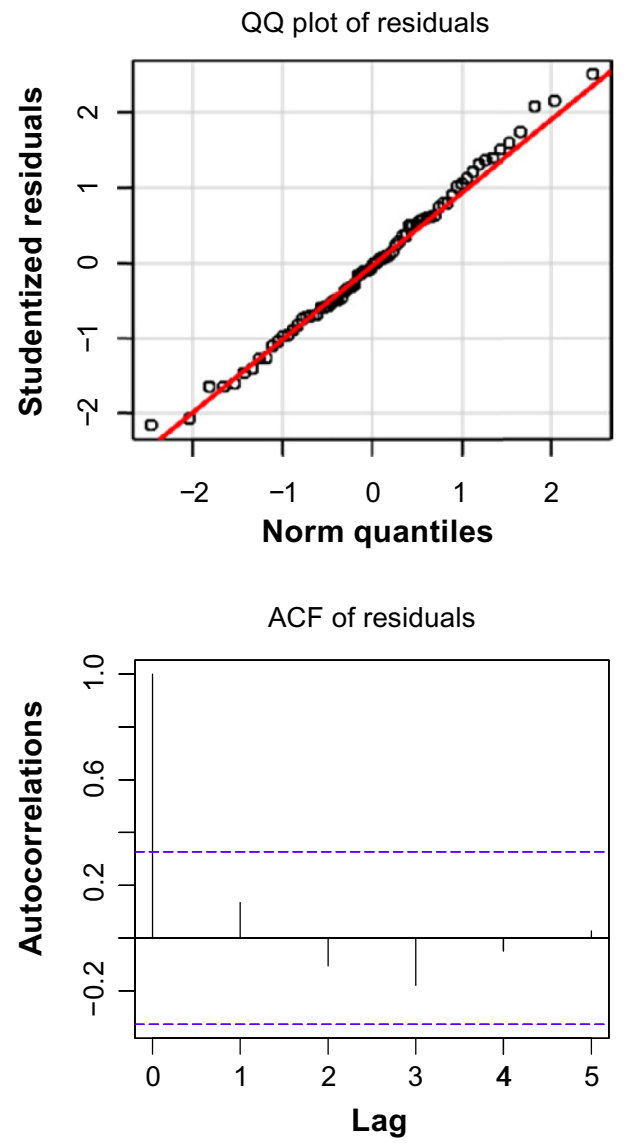

Figure 3 Fit and validation of the log-linear model for RL cells.

Abbreviations: RL cells, American Type Culture Collection CRL-226I cells; ACF, autocorrelation function. 
Table 2 Estimations for the logarithm of THP-I cell counts

\begin{tabular}{llll}
\hline Coefficient & Estimate & SE & $P$-value \\
\hline$a$ & 12.37 & $5 \times 10^{-2}$ & $<2 \times 10^{-16}$ \\
$b$ & 0.0212 & $4 \times 10^{-4}$ & $<2 \times 10^{-16}$ \\
$c$ & $5 \times 10^{-6}$ & $7 \times 10^{-7}$ & $8.6 \times 10^{-11}$ \\
\hline
\end{tabular}

Abbreviations: SE, standard error; THP-I cells, American Type Culture Collection TIB-202 cells.

estimated coefficients. Figure 4 presents the residual analysis. In contrast with the RL case, the curvature $c$ is significantly positive $\left(P=8.6 \times 10^{-11}\right)$. The proportion of the variation of $Y_{i k}$ explained by the fitted model is excellent $\left(R^{2}=0.99\right)$. The $95 \%$ confidence interval of the mean THP-1 growth rate $b$ is [0.0204, 0.0227]. This corresponds to a doubling time between 30.5 and 34 hours, ie, slightly below the values given in Tsuchiya et $\mathrm{al}^{9}$ (35-50 hours) and above those of Tsuchiya et $\mathrm{al}^{26}$ (24-30 hours).

The QQ plot of residuals (Figure 4) is close to linear, and the plot of residuals versus time does not show any misspecification of the non-random part or a heteroscedasticity problem. The Durbin-Watson test $(P=0.16)$ and the runs test $(P=0.75)$ indicate no violation of the hypothesis of
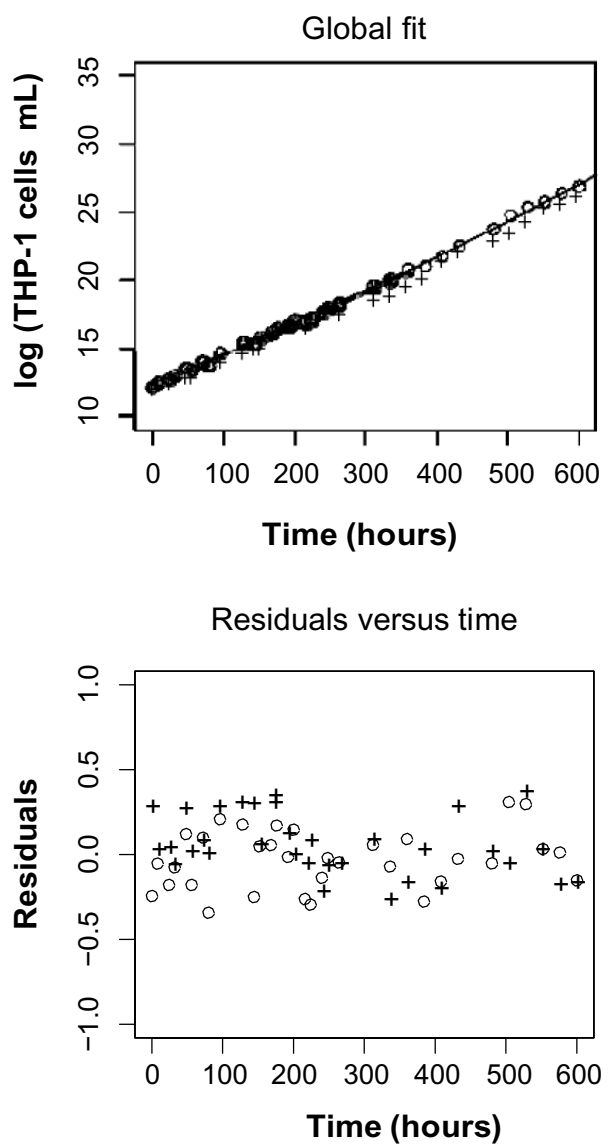

error independence. As expected, the growth rate of THP-1 is significantly smaller than that of RL $(P<0.0001)$.

\section{Simultaneous growth of RL and THP-I}

Let $Y_{i j k}$ denote the logarithm of the ratio of RL to THP-1 cell counts at time $t_{k}$, where $i$ denotes the replicate $(i=1,2,3)$ and $j$ the initial nominal value of the ratio, which will be called "dilution". Indices $j=1,2,3$ correspond to dilutions of $0.5 \%, 1 \%$, and $5 \%$, respectively. Four different models were considered:

$$
\begin{gathered}
Y_{i j k}=a+a_{j}+b t_{k}+\varepsilon_{i j k} \\
Y_{i j k}=a+a_{j}+b t_{k}+b_{j} t_{k}+\varepsilon_{i j k} \\
Y_{i j k}=a+a_{j}+b t_{k}+b_{j} t_{k}+c t_{k}^{2}+\varepsilon_{i j k} \\
Y_{i j k}=a+a_{j}+b t_{k}+c t_{k}^{2}+c_{j} t_{k}^{2}+\varepsilon_{i j k} \\
Y_{i j k}=a+a_{j}+b t_{k}+b_{j} t_{k}+c t_{k}^{2}+c_{j} t_{k}^{2}+\varepsilon_{i j k}
\end{gathered}
$$

Model (M0) is the simplest: the expected $\log$ ratio $Y_{i j k}$ is modeled by a straight line, the slope $b$ of which does not depend on dilution. In model (M1), the slopes $b+b j$ may depend on dilution. In the actual fit, the slopes are found to
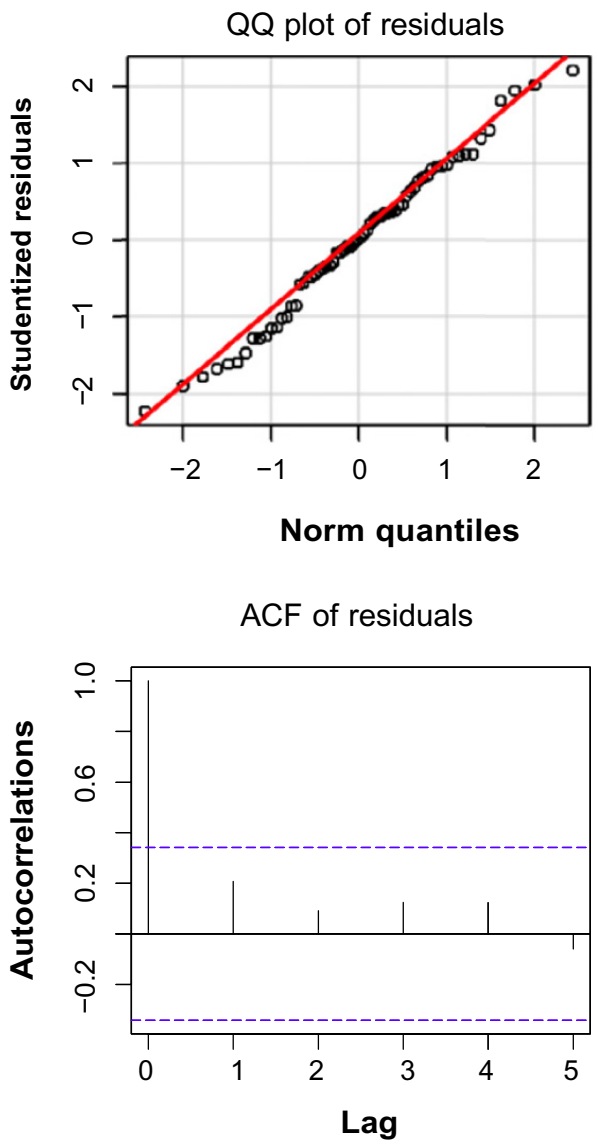

Figure 4 Fit and validation of the log-quadratic model for THP-I cells.

Abbreviations: THP-I cells, American Type Culture Collection TIB-202 cells; ACF, autocorrelation function. 
Table 3 Tests of embedded models for the log ratio of $R L$ versus THP-I cell counts

\begin{tabular}{llll}
\hline Embedded models & df & $\boldsymbol{F}$ & $\boldsymbol{P}$-value \\
\hline (M3) versus (M2b) & $(2,29 I)$ & 24.67 & $1.3 \times 10^{-10}$ \\
(M2b) versus (MI) & $(I, 293)$ & 22.8 & $8 \times 10^{-39}$ \\
(MI) versus (M0) & $(2,294)$ & 118 & $1.8 \times 10^{-38}$ \\
(M3) versus (M2c) & $(2,29 I)$ & 2.68 & 0.0705 \\
\hline
\end{tabular}

Abbreviations: df, degrees of freedom; RL cells, American Type Culture Collection CRL-226I cells; THP-I cells, American Type Culture Collection TIB-202 cells.

decrease as the initial proportion of RL increases. This is consistent with Figure 1. However, it hides the relevance of the quadratic models. Model (M3) is the complete logquadratic model: both the slopes $b+b_{j}$ and the curvatures $c+c$ may depend on dilution. Models (M2b) and (M2c) are embedded into (M3): in model (M2b), the slope does not depend on dilution and in model (M2c) the curvature does not depend on dilution.

For all four models, the linear fit was computed, and pairs of embedded models were then tested by Fisher's test of analysis of variance. The results are shown in Table 3: the degrees of freedom (df), the Fisher's test statistic $F$, and the ANOVA $P$-values are given. The conclusions are the following. The first three comparisons are significant, ie, the bigger model is better than the embedded one, and the (M3) versus (M2c) comparison is not. The conclusion of the four comparisons is that the best fitted model is (M2c). This indicates that if curvatures are included in the model, the slopes do not depend significantly on dilution. This is consistent with the theoretical derivation of the log-quadratic model (7). In model (M2c) the estimated slope is $\hat{b}=6.1 \times 10^{-3}$ and the $95 \%$ confidence interval on $b$ is $\left[5.6 \times 10^{-3}, 6.6 \times 10^{-3}\right]$. Recall from (7) that the slope $b$ of (M2c) should be understood as the difference between the slopes of models (9) for RL and THP-1. From the two previous sections, the estimated difference is $1.01 \times 10^{-2}$, which is above the confidence interval on $b$ in (M2c). Next, we tested the three pairwise differences of curvatures $c_{j}$ in the accepted model (M2c). The results are presented in Table 4, and the value of the Student's $t$-test statistic and the $P$-value are given (degrees of freedom, 293). All three differences are significant. The estimated values of the $c+c_{j}$ curvatures are given in Table 5 . It turns out that

Table 4 Pairwise tests for differences in cj curvatures in model (M2c)

\begin{tabular}{lll}
\hline Null hypothesis & $\boldsymbol{t}$ & $\boldsymbol{P}$-value \\
\hline$c_{1}=c_{2}$ & 2.4 & 0.016 \\
$c_{1}=c_{3}$ & 17.9 & $6.3 \times 10^{-49}$ \\
$c_{2}=c_{3}$ & 16.1 & $4.5 \times 10^{-42}$ \\
\hline
\end{tabular}

Table 5 Estimated c + cj curvatures in model $(\mathrm{M} 2 \mathrm{c})$

\begin{tabular}{ll}
\hline Dilution $j$ & $c+c_{j}$ \\
\hline$j=I(0.5 \%)$ & $5.3 \times 10^{-6}$ \\
$j=2(1 \%)$ & $4.96 \times 10^{-6}$ \\
$j=3(5 \%)$ & $1.6 \times 10^{-6}$ \\
\hline
\end{tabular}

$c_{1}>c_{2}>c_{3}$. Therefore, the curvature $c+c_{j}$ decreases as the initial proportion of $\mathrm{RL}$ increases. This phenomenon is similar to that observed in Figure 1. Indeed, when the curvatures are neglected (model [M1]), the slopes were found to be decreasing as the initial proportion of RL increases. The theoretical explanation is given by the mathematical model. Model (M1) amounts to keeping only the first term in the Taylor expansion (5). Since the next term is positive, the adjusted values of the slopes increase with time. Model (M2c) considers the first two terms in (5), neglecting the third one. If that neglected term is positive, then the same effect will occur, ie, adjusted values of curvatures increase with time. The third term is proportional to the skewness of $B$. Observing decreasing curvatures as in Table 5 is an indication that the skewness of $B$ may be positive, where $B$ is the (random) difference in growth rate between RL and THP-1. Koutsoumanis and Lianou $^{5}$ have proposed a logistic distribution as a model for variability in growth rate. That distribution has null skewness. We conjecture that distributions with positive skewness provide better models for variable growth rates.

\section{Conclusion}

It is a well-known fact that unchecked populations grow exponentially fast, and this is backed up by countless experiments that validate the mathematical theory of branching processes. ${ }^{4}$ Any clone stemming from a single cell can be associated with an exponential growth rate, also called the Malthusian parameter. It can be seen as the slope, over a large period of time, of the line fitting logarithms of the number of cells against time. What is questioned here is the idea that clones stemming from different cells in a given strain should have the same growth rate. Unlike Koutsoumanis and Lianou ${ }^{5}$ or Tomelleri et al, ${ }^{11}$ we do not provide direct evidence for the intrinsic variability of growth rates, but instead indirect proof coming from a simultaneous growth experiment. ${ }^{10}$

This entailed growing two cancer cell lines, ie, $\mathrm{RL}^{8}$ and THP- $1,{ }^{9}$ in the same vessels. If a single growth rate existed for all RL clones and another for all THP-1 clones, then the ratio should grow exponentially, the rate being the difference of the two growth rates. In that case, the growth rate of the ratio should not depend on the initial proportions of RL versus THP-1. Our observations disproved this, ie, the growth rate of the ratio was 
found to increase as the initial proportion of RL decreased (Figure 1). Assuming that growth rates may vary among clones provides both intuitive and theoretical explanations.

The intuitive explanation is as follows. Consider a growth rate as attached to each cell of a given clone. If clones grow at different rates, the proportion of cells in faster growing clones will gradually increase. In other words, the distribution of growth rates at increasing times will be shifted toward larger values. This explains why, when the initial proportion of RL cells is $0.5 \%$, the population of RL contains more rapid breeders at the time it reaches $5 \%$ than at time 0 . Therefore, the (apparent) growth rate for an initial proportion of $0.5 \%$ is larger than for an initial proportion of $5 \%$.

The theoretical explanation is as follows. If growth rates of different clones are considered as independent random variables with a positive variance, then the model fitting the logarithms of cell numbers against time must contain a quadratic term proportional to the variance of growth rates: variable growth rates imply that higher-order terms must be added to the classical log-linear model. Now if a population grows according to a log-quadratic model, and a log-linear model is fitted instead, then the estimated slope over an interval of time should increase as the interval moves to the right (see Figure 2). This will overestimate the mean growth rate.

To validate our theoretical explanation, we had to compare the fits of the log-linear and log-quadratic models to our experimental data. For the separate growth data, the log-linear model was better than the log-quadratic model for RL, but the reverse was true for THP-1. The log-quadratic model clearly provided a better fit for simultaneous growth data. This may seem paradoxical; indeed, the same model should be adopted for separate and simultaneous growths. The explanation of this apparent contradiction is statistical. The estimated curvature terms are in all cases smaller by several orders of magnitude than the estimated slopes. Therefore, the log-linear and log-quadratic models can hardly be distinguished when the cell counts range over several orders of magnitude, as in separate growths. This cannot be the case for simultaneous growth data, where the ratios range from a few percent to $100 \%$. We believe that if more values were collected at the beginning in a separate growth experiment, then the logquadratic model would provide a better fit.

There remains the issue of a probabilistic model to be fit on variable growth rates. Our derivation of the cumulant generating function shows that classical models of positive random variables, such as gamma, log-normal, or logistic distributions, ${ }^{5}$ cannot be used here. Indeed, their exponentially decaying tail implies that the equivalent growth rate would become infinite at a finite time, which is not realistic. Therefore, a truncated model would have to be used instead. In any case, the chosen distribution would have to by adjusted to real data. Ideally, these data should be collected from the observation of colony growth of individual cells, as reported by Koutsoumanis and Lianou for Salmonella enterica ${ }^{5}$ or by Tomelleri et $\mathrm{al}^{11}$ for leukemia cells. This will be the objective of future work.

\section{Acknowledgments}

This work was supported by Laboratoire d'Excellence TOUCAN (Toulouse Cancer). The authors are indebted to the anonymous referees for their important suggestions.

\section{Disclosure}

The authors report no conflicts of interest in this work.

\section{References}

1. Chevin LM. On measuring selection in experimental evolution. Biol Letters. 2011;7(2):210-213.

2. Manna F, Galet R, Martin G, Lenormand T. The high-throughput yeast deletion fitness data and the theories of dominance. J Evolutionary Biol. 2012;25(5):892-903.

3. Powell EO. Growth rate and generation time of bacteria with special reference to continuous culture. J General Microbiol. 1956; 15(3):492-511.

4. Kimmel M, Axelrod D. Branching Processes in Biology. SpringerVerlag, New York, 2002.

5. Koutsoumanis KP, Lianou A. Stochasticity in colonial growth dynamics of individual bacterial cells. Appl Environ Microbiol. 2013;79(7):2294-2301.

6. Deenick EK, Gett AV, Hodgkin PD. Stochastic model of T-cell proliferation: a calculus revealing IL-2 regulation of precursor frequencies, cell cycle time, and survival. J Immunology. 2003;170:4963-4972.

7. Zilman A, Ganusov VV, Perelson AS. Stochastic models of lymphocyte proliferation and death. PLoS One. 2010;5(9):e12775.

8. Beckwith M, Longo DL, O'Connell CD, Moratz C, Urba WJ. Phorbol ester-induced, cell-cycle-specific, growth inhibition of human b-lymphoma cell lines. J Nat Cancer Institute. 1990;82(6):501-509.

9. Tsuchiya S, Yamabe M, Yamaguchi Y, Kobayashi Y, Konno T, Tada K. Establishment and characterization of a human acute monocytic leukemia cell line (THP-1). Int J Cancer. 1980;26(2):171-176.

10. Dykhuizen DE. Experimental studies of natural selection in bacteria. Annu Rev Ecol Syst. 1990;21:373-398.

11. Tomelleri C, Milotti E, Dalla Pellegrina C, et al. A quantitative study of growth variability of tumour cell clones in vitro. Cell Proliferation. 2008;41(1):177-191.

12. Novick A. Growth of bacteria. Annual Review of Microbiology. 1955;9:97-110.

13. Zwietering MH, Jongenburger I, Rombouts FM, van't Riet K. Modeling of the bacterial growth curve. Appl Environ Microbiol. 1990;56(6):1875-1881.

14. Peleg M, Corradini MG. Microbial growth curves: what the models tell us and what they cannot. Crit Rev Food Sci Nutr. 2011;51(10):917-945.

15. Brook RF. Variability in the cell cycle and control of proliferation. In John PC, editor, The Cell Cycle, pages 35-62. Cambridge University Press, 1981.

16. Delignette-Muller M-L. Relation between the generation time and the lag time of bacterial growth kinetics. International Journal of Food Microbiology. 1998;43(1):97-104. 
17. Baty F, Delignette-Muller M-L. Estimating the bacterial lag time: which model, which precision? International Journal of Food Microbiology. 2004;91(3):261-277.

18. Hansen TF. Selection in asexual populations - an extension of the fundamental theorem. $J$ Theor Biol. 1992;155(4):537-544.

19. Harris TE. The Theory of Branching Processes. Springer-Verlag, Berlin, 1963.

20. Athreya KB, Ney PE. Branching Processes. Springer-Verlag, Berlin, 1972.

21. Mair P. Interpreting Standard and Non Standard Log-Linear Models. Waxmann, Münster, 2006.

22. von Eye A, Mun EY. Log-Linear Modeling: Concepts Interpretation, and Application. Wiley, New York, 2013.
23. Kendall MG, Stuart A. The Advanced Theory of Statistics: Distribution Theory. Wiley-Blackwell, New York, 6th edition, 1994.

24. Dembo A, Zeitouni O. Large Deviations Techniques and Applications. Springer, New York, 2nd ed, 1998.

25. Stone G, Chapman B, Lovell D. Development of a log-quadratic model to describe microbial inactivation, illustrated by thermal inactivation of Clostridium botulinum. App Environ Microbiol. 2009;75(22) 6998-7005.

26. Tsuchiya S, Minegishi N, Minegishi M, Sato T, Konno T. Adaptation of a human monocytic leukemia cell line (THP-1) in a protein-free chemically defined medium. Tohoku J Exp Med. 1986;150(4):455-465.

Open Access Medical Statistics

\section{Publish your work in this journal}

Open Access Medical Statistics is an international, peer- reviewed, open access journal publishing original research, reports, reviews and commentaries on all areas of medical statistics. The manuscript management system is completely online and includes a very quick and fair

peer-review system. Visit http://www.dovepress.com/testimonials.php to read real quotes from published authors. 